\title{
Structural effects of different NH-acids and dialkyl acetylenedicarboxylates on the kinetic parameters of stable phosphorus ylids: a mechanistic investigation
}

\author{
Mohammad Zakarianejad $^{\mathrm{a}}$, Sayyed Mostafa Habibi-Khorassani ${ }^{\text {** }}$, Batoul Makiabadi ${ }^{\mathrm{c}}$ and \\ Aboozar Taheri ${ }^{\mathrm{d}}$
}

${ }^{a}$ Department of Chemistry, Payam Noor University, Tehran, I. R. Iran

${ }^{b}$ Department of Chemistry, the University of Sistan and Baluchestan, Zahedan, Iran

${ }^{c}$ Department of Chemical Engineering, Sirjan University of Technology, Sirjan, Iran

${ }^{d}$ Department of Chemistry, Lamerd Branch, Islamic Azad University, Lamerd, Iran

\section{H R O N I C L E}

Article history:

Received October 25, 2012

Received in Revised form

December 6, 2012

Accepted 22 February 2013

Available online

23 February 2013

Keywords:

Stable phosphorus ylides

Dialkyl acetylenedicarboxylates

NH-acid

Heterocyclic compounds

Kinetic studies

\begin{abstract}
A B S T R A C T
Kinetic studies were made of the reactions between triphenylphosphine $\mathbf{1}$ and dialkyl acetylenedicarboxylates 2 , in the presence of NH-acids, such as benzotriazole, 5methylbenzotriazole or 5-chlorobenzotriazole 3 (as a protic/nucleophilic reagent). To determine the kinetic parameters of the reactions, they were monitored by UV spectrophotometry. Useful information was obtained from studies of the effect of solvent, structure of reactants (dialkyl acetylenedicarboxylates and $\mathrm{NH}$-acids) and also concentration of reactants on the reaction rates. First and third steps $\left(k_{2}, k_{3}\right)$ of all reactions were recognized as a rate determining and fast steps, respectively. Proposed mechanism was confirmed on the basis of experimental data.
\end{abstract}

\section{Introduction}

Organophosphorus compounds have emerged as important reagents and intermediates in organic synthesis $^{1}$. An important group of this class is phosphorus ylides, which have been used in many reactions and synthesis of organic compounds ${ }^{2-29}$. The prominent role of these compounds is to convert the carbonyl groups to carbon-carbon double bonds ${ }^{7}$. From the large number of methods available for the synthesis of phosphorus ylides, the most important one involves the reaction of a * Corresponding author. Tel: +985412446565; Fax: +985412446565 
phosphonium salt with a base ${ }^{14,20}$. In recent years a method has been developed for the preparation of this family by using a novel approach employing vinyl phosphonium salts ${ }^{15,16}$. The phosphonium salts are most often converted to the ylides by treatment with a strong base, though weaker bases can be used if the salt is acidic enough. Michael addition of phosphorus (ш) compounds such as triphenylphosphine to acetylenic esters leads to reactive 1,3-dipolar intermediate betaines which are not detected even at low temperatures ${ }^{17}$. These unstable species can be trapped by a protic reagent, $\mathrm{ZH}$, such as methanol, amide, imide, etc. to produce various compounds e.g. ylides $^{5-29}$. A facile reaction between triphenylphosphine $\mathbf{1}$, dialkyl acetylenedicarboxilates $\mathbf{2}(\mathbf{2 d}, \mathbf{2 e}$ or $\mathbf{2 f})$ and imidazole, 2-methylimidazole or 4-methylimidazole $\mathbf{3}$ (3a, 3b or 3c) (as $\mathrm{NH}$-acid) has been reported earlier $^{30}$, but the kinetic studies of this reaction has not been investigated yet. In order to gain further insight into the reaction mechanism, a kinetic study of the reaction between compounds $\mathbf{1}, \mathbf{2}$ and $\mathbf{3}$ as $\mathrm{NH}$-acid was undertaken by the UV spectrophotometeric technique. On the basis of the well established chemistry of trivalent phosphorus nucleophiles, ${ }^{1-3}$ it is reasonable to assume that phosphorus ylide 4 results from the initial addition of triphenylphosphine to the acetylenic ester 2 (2d, 2e or 2f) (rate constant $k_{2}$ ) and subsequent protonation of the 1:1 adduct by the NH-acid 3 (3a, 3b or 3c) (rate constant $k_{3}$ ) to form phosphoranes 4 (rate constant $k_{4}$ ). To gain further insight into the reaction mechanism between triphenylphosphin 1, dialkyl acetylenedicarboxylates $\mathbf{2}$ and $\mathbf{3}$ (3a, 3b or 3c) (as NH- heterocyclic compound) for generation of phosphorus ylides $\mathbf{4}$ (Scheme 1), a kinetic study of the reactions was undertaken by UV spectrophotometeric technique.

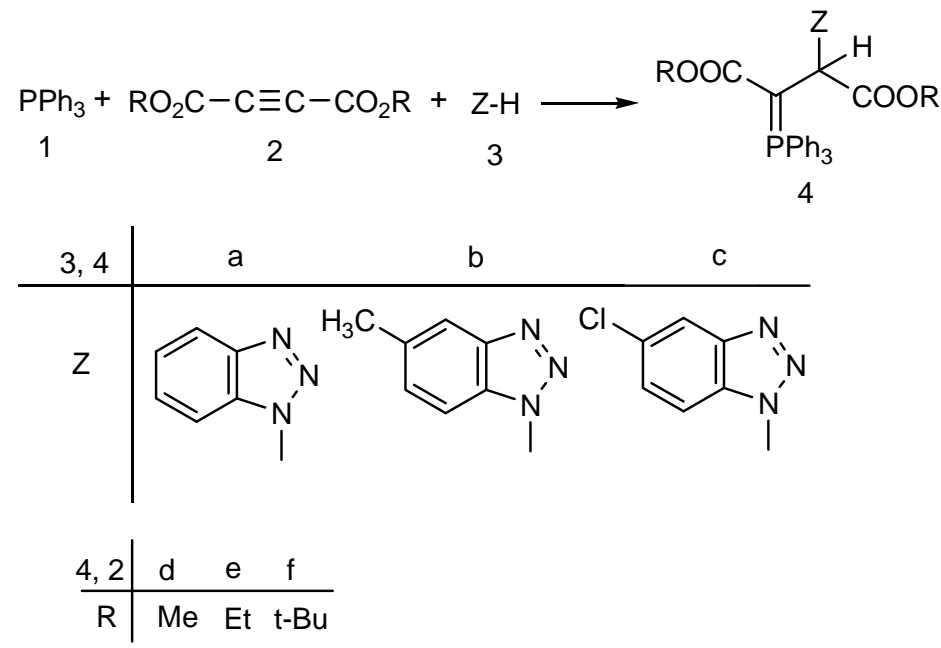

Scheme 1

\section{Experimental}

\subsection{Chemicals and Apparatus Used}

Dialkyl acetylenedicarboxylates, triphenylphosphine, benzotriazole, 5-methylbenzotriazole and 5chlorobenzotriazole were purchased from Fluka (Buchs, Switzerland) and used without further purifications. All extra pure solvents including the 1,4-dioxan and 1,2-dichloroethane were obtained from Merck (Darmstadt, Germany). A Cary UV-Vis spectrophotometer model Bio-300 with a $10 \mathrm{~mm}$ light-path quartz spectrophotometer cell was employed throughout the current work.

\subsection{Method}

A first, it was necessary to find the appropriate wavelength to follow the kinetic study of the reaction. For this purpose, in the first experiment, $3 \times 10^{-3} \mathrm{M}$ solutions of compounds $\mathbf{1}$, 2f and 3a were prepared 
in 1,4-dioxan. An approximately $3 \mathrm{~mL}$ aliquot from each reactant was pipetted into a $10 \mathrm{~mm}$ light path quartz spectrophotometer cell, and the relevant spectra were recorded over the wavelength range of 190-400 nm. Figures 1, 2 and $\mathbf{3}$ show the ultraviolet spectra of compounds 1, 2f and 3a respectively. In a second experiment, a $1 \mathrm{~mL}$ aliquot from the $3 \times 10^{-3} \mathrm{M}$ solutions of each compound of 1 and 3a was pipetted first into a quartz spectrophotometer cell (as there is no reaction between them), later $1 \mathrm{~mL}$ aliquot of the $3 \times 10^{-3} \mathrm{M}$ solution of reactant $2 \mathrm{f}$ was added to the mixture and the reaction monitored by recording scans of the entire spectra every $11 \mathrm{~min}$ at ambient temperature. The ultra-violet spectra shown in Figure 4 are typical. From this, the appropriate wavelength was found to be $330 \mathrm{~nm}$ (corresponding mainly to product 4), because at this wavelength, compounds $\mathbf{1}, \mathbf{2} \mathbf{f}$ and 3a have relatively no absorbance value. This, then provided the opportunity to fully investigate the kinetics of the reaction between triphenylphosphine 1, di-tert-butyl acetylenedicarboxylate $\mathbf{2 f}$ and benzotriazole $3 \mathbf{a}$ at $330 \mathrm{~nm}$ in the presence of 1, 2-dichloroethaneas solvent. Since the spectrophotometer cell of the UV instrument had a $10-\mathrm{mm}$ light-path cuvette, the UV-Vis spectra of compound 4c were measured over the concentration range $\left(2 \times 10^{-4} \mathrm{M} \leq \mathrm{M}_{4 \mathrm{c}} \leq 10^{-3} \mathrm{M}\right)$ to check for a linear relationship between absorbance values and concentrations. With the suitable concentration range and wavelength identified, the following procedure was employed.

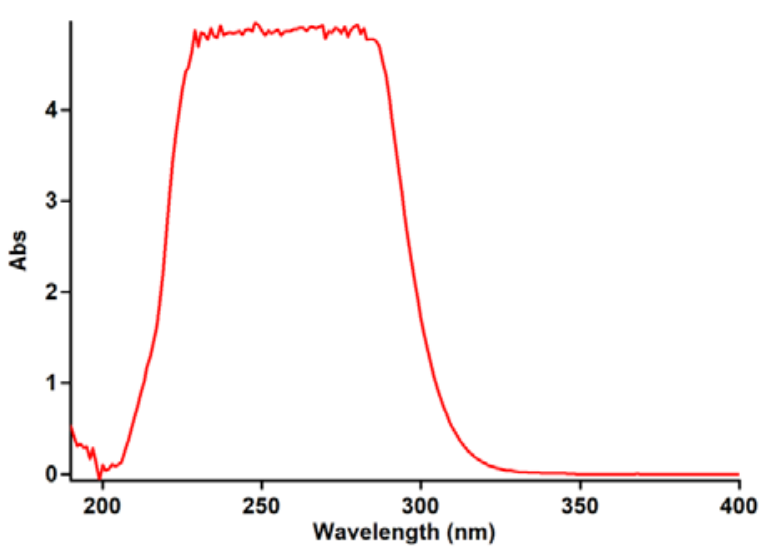

Fig. 1. The UV spectrum of $10^{-3} \mathrm{M}$ triphenylphosphine 1 in 1,4-dioxan

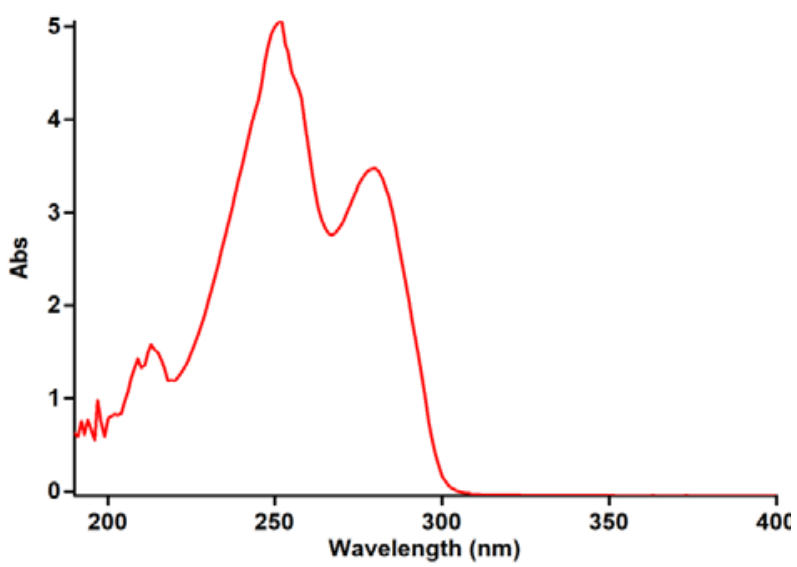

Fig. 3. The UV spectrum of $10^{-3} \mathrm{M}$ benzotriazole 3a in 1,4-dioxan

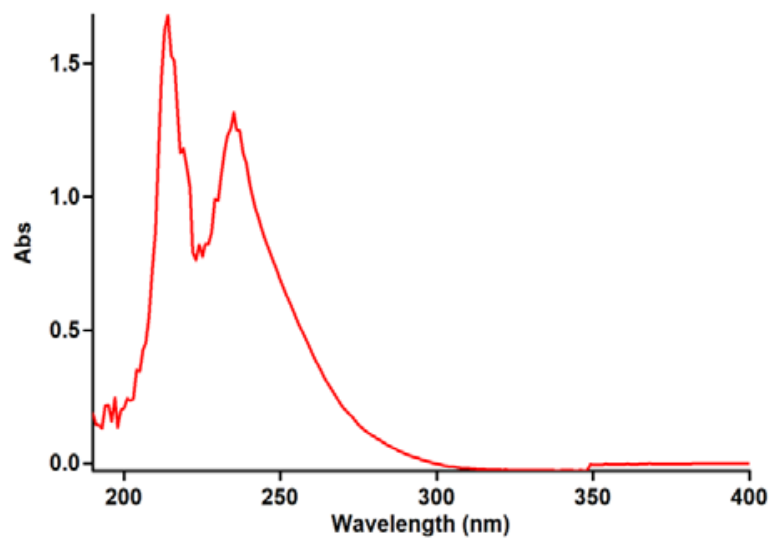

Fig. 2. The UV spectrum of $10^{-3} \mathrm{M}$ di-tert-butyl acetylenedicarboxylate $\mathbf{2 f}$ in 1,4-dioxan

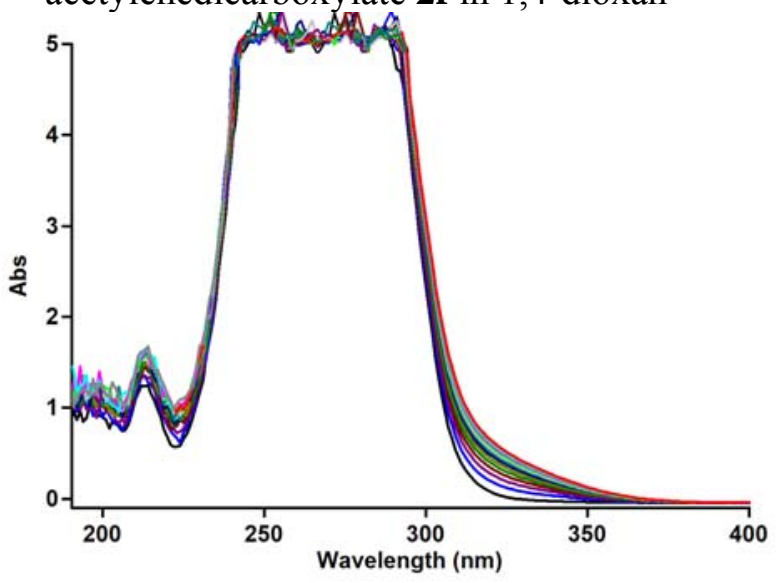

Fig. 4. The UV spectra of the reaction between $10^{-3} \mathrm{M}$ of each compounds $\mathbf{1}, \mathbf{2} \mathbf{f}$ and $\mathbf{3 a}$ as reaction proceeds in 1,4-dioxan 
For each kinetic experiment, first a $1 \mathrm{~mL}$ aliquot from each freshly made $3 \times 10^{-3} \mathrm{M}$ solution of compounds 1 and 3 in 1,4-dioxan was pipetted into a quartz cell, and then a $1 \mathrm{~mL}$ aliquot of the $3 \times 10^{-}$ ${ }^{3} \mathrm{M}$ of solution of reactant $\mathbf{2} \mathbf{f}$ was added to the mixture, keeping the temperature constant at $12.0{ }^{\circ} \mathrm{C}$. The reaction kinetic was followed by plotting UV absorbance against time. Figure 5 shows the absorbance change (dotted line) versus time for the 1:1:1 addition reaction between compounds $\mathbf{1}, \mathbf{2 f}$ and 3a at $12.0{ }^{\circ} \mathrm{C}$. The infinity absorbance $\left(\mathrm{A}_{\infty}\right)$ that is the absorbance at reaction completion, can be obtained from Figure 5 at $\mathrm{t}=160 \mathrm{~min}$. With respect to this value, zero, first or second curve fitting could be drawn automatically for the reaction by the software ${ }^{31}$ associated with the UV instrument. Using the original experimental absorbance versus time data provided a second-order fit curve (full line) that fits exactly the experimental curve (dotted line) as shown in Figure 6. Thus, the reaction between triphenylphosphine 1, di-tert-butyl acetylenedicarboxylate $\mathbf{2 f}$ and 3a follows second-order kinetics. The second-order rate constant $\left(k_{2}\right)$ is then automatically calculated using a standard equation $^{31}$ within the program at $12.0^{\circ} \mathrm{C}$. It is reported in Table $\mathbf{1}$.

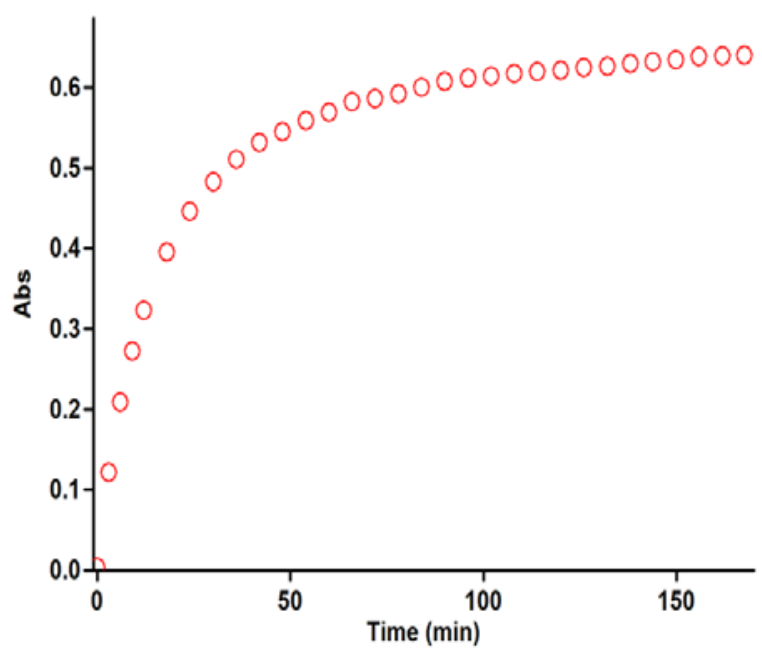

Fig. 5. Experimental absorbance change (dotted line) against time at $330 \mathrm{~nm}$ for the reaction between compounds $\mathbf{1}, \mathbf{2 f}$ and $3 \mathbf{a}$ at $12.0^{\circ} \mathrm{C}$ in 1,4-dioxan

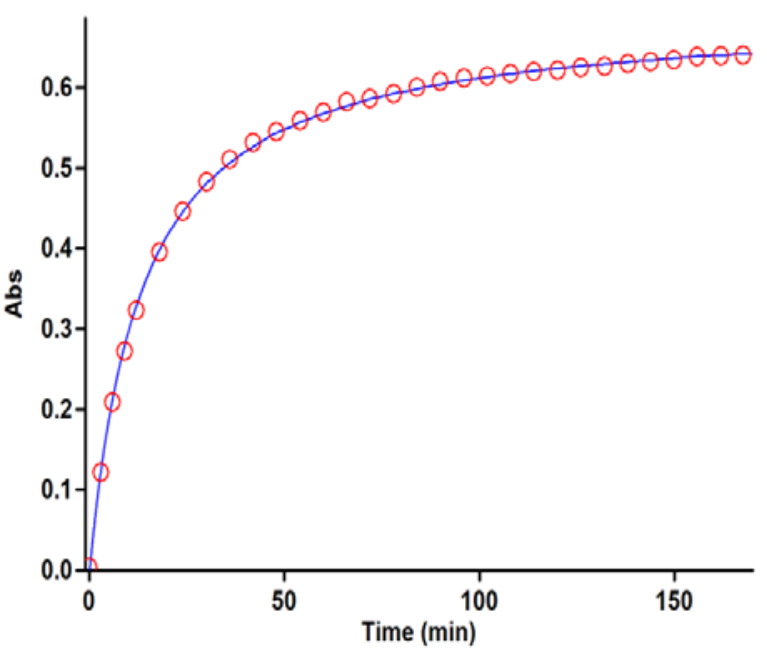

Fig. 6. Second order fit curve (solid line) accompanied by the original experimental curve (dotted line) for the reaction between 1, 2f and 3a at $330 \mathrm{~nm}$ and $12.0^{\circ} \mathrm{C}$ in 1,4-dioxan

Furthermore, kinetic studies were carried out using the same concentration of each reactant in the continuation of experiments with concentrations of $5 \times 10^{-3} \mathrm{M}$ and $7 \times 10^{-3} \mathrm{M}$ respectively. As expected, the second-order rate constant was independent of concentration and its value was the same as in the previous experiment. In addition, the overall order of reaction was also 2.

\subsection{Effect of solvents and temperature}

To determine the effect of change in temperature and solvent environment on the rate of reaction, it was elected to perform various experiments at different temperatures and solvent polarities but otherwise under the same conditions as for the previous experiment. For this purpose, 1,2dichloroethane with dielectric constant 10.3 respectively were chosen as a suitable solvent since it is not only could be dissolved all compounds but also did not react with them. The effects of solvents and temperature on the rate constant are given in Table $\mathbf{1}$. The results show that the rate of reaction in each case was increased at higher temperature. In addition, the rate of reaction between $\mathbf{1}, \mathbf{2} \mathbf{f}$ and $3 \mathbf{a}$ was accelerated in a higher dielectric constant environment (1,2-dichloroethane) in comparison with a lower dielectric constant environment (1,4-dioxan) at all temperatures investigated. In the temperature range studied, the dependence of the second-order rate constant $\left(\ln k_{2}\right)$ of the reactions on reciprocal temperature is consistent with the Arrhenius equation. 
Table 1 The values of second order rate constant for the three reactions $(\mathbf{1}, \mathbf{2 f}$ and $\mathbf{3 a}),(\mathbf{1}, \mathbf{2} \mathbf{f}$ and $\mathbf{3 b})$ and $(\mathbf{1}, \mathbf{2} \mathbf{f}$ and $\mathbf{3 c})$ in the presence of different solvents at all temperatures investigated

\begin{tabular}{|c|c|c|c|c|c|c|}
\hline \multirow{2}{*}{3} & \multirow{2}{*}{ Solvent } & \multirow{2}{*}{$\varepsilon$} & \multicolumn{4}{|c|}{$k_{2} \cdot \mathbf{M}^{-1} \cdot \min ^{-1}$} \\
\hline & & & $12.0^{\circ} \mathrm{C}$ & $17.0^{\circ} \mathrm{C}$ & $22.0^{\circ} \mathrm{C}$ & $27.0^{\circ} \mathrm{C}$ \\
\hline \multirow{2}{*}{ 3a } & 1,4-dioxan & 2.02 & 88.4 & 111.8 & 142.0 & 173.7 \\
\hline & 1,2-dichloroethane & 10.3 & 109.3 & 134.2 & 163.5 & 196.4 \\
\hline \multirow{2}{*}{$3 \mathbf{b}$} & 1,4-dioxan & 2.02 & 80.2 & 104.4 & 132.1 & 162.9 \\
\hline & 1,2-dichloroethane & 10.3 & 101.6 & 126.4 & 153.1 & 184.7 \\
\hline \multirow{2}{*}{ 3c } & 1,4-dioxan & 2.02 & 103.2 & 122.6 & 149.1 & 180.0 \\
\hline & 1,2-dichloroethane & 10.3 & 126.6 & 147.7 & 172.8 & 206.5 \\
\hline
\end{tabular}

\subsection{Effect of concentration}

To determine reaction order with respect to triphenylphosphine $\mathbf{1}$ and dialkyl acetylenedicarboxylate $\mathbf{2}$ (2f), in the continuation of experiments, all kinetic studies were carried out in the presence of excess 3 . Under this condition, the rate equation may therefore be expressed as:

rate $=k_{o b s}[1]^{\alpha}[2]^{\beta} \quad k_{o b s}=k_{2}[3]^{\gamma}$ or $\ln k_{o b s}=\ln k_{2}+\gamma \ln [3]$

In this case $\left(3 \times 10^{-2} \mathrm{M}\right.$ of 3 instead of $\left.3 \times 10^{-3} \mathrm{M}\right)$ using the original experimental absorbance versus time data provides a second order fit curve (full line) against time at $330 \mathrm{~nm}$ which exactly fits the experimental curve. The value of rate constant was the same as that of obtained from the previous experiment $\left(3 \times 10^{-3} \mathrm{M}\right)$. Repetition of the experiments with $5 \times 10^{-2} \mathrm{M}$ and $7 \times 10^{-2} \mathrm{M}$ of 3 gave, separately, the same fit curve and rate constant. In fact, the experimental data indicated that the observed pseudo second order rate constant $\left(k_{\text {obs }}\right)$ was equal to the second order rate constant $\left(k_{2}\right)$, this is possible when $\boldsymbol{\gamma}$ is zero in equation (1). Therefore, It appears that the reaction is zero order with respect to 3 (protic/nucleophilic reagent) and second order with sum of $\mathbf{1}$ and $\mathbf{2}(\mathbf{2 f})(\alpha+\beta=2)$, respectively.

To determine reaction order with respect to dialkyl acetylenedicarboxylate $\mathbf{2}(\mathbf{2 f})$, the continuation of experiment was performed in the presence of excess of 1 (rate $=k_{\text {obs }}^{\prime}[3]^{\gamma}[2]^{\beta}, k^{\prime}{ }_{\text {obs }}=k_{2}$ $\left.[1]^{\alpha}(2)\right)$. The original experimental absorbance versus time data and provide a pseudo first order fit curve at $330 \mathrm{~nm}$, which exactly fits the experimental curve (dotted line) as shown in Fig. 7.

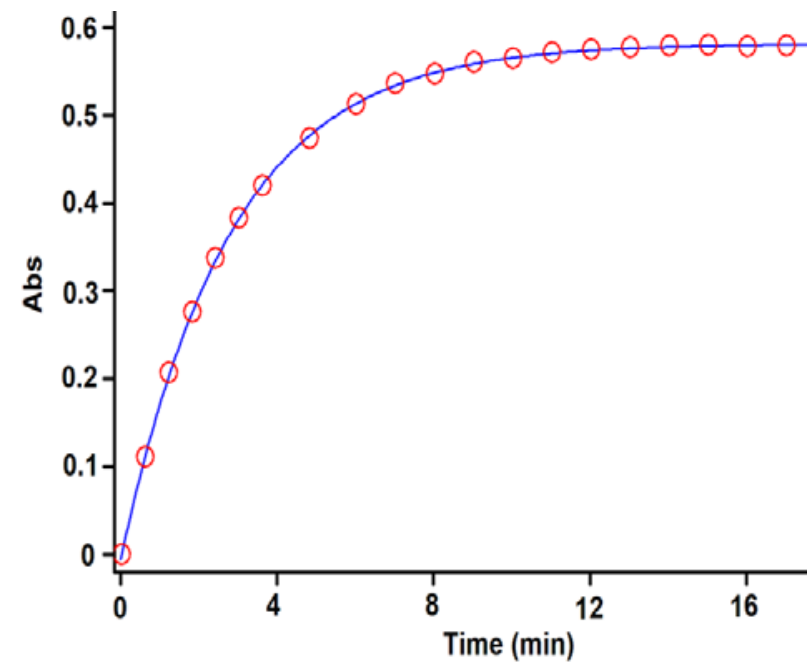

Fig. 7. Pseudo first order fit curve (solid line) for the reaction between $\mathbf{2 f}$ and $3 \mathbf{a}$ in the presence of excess $1\left(10^{-2} \mathrm{M}\right)$ at $330 \mathrm{~nm}$ and $12.0{ }^{\circ} \mathrm{C}$ in 1,4-dioxan. 
As a result since $\gamma=0$ (as determined previously), it is reasonable to accept that the reaction is first order with respect to compound $2(\mathbf{2 f})(\beta=1)$. Because the overall order of reaction is $2(\alpha+\beta+$ $\gamma=2)$ it is obvious that $\alpha=1$ and order of triphenylphosphine 1 must be equal to one. This observation was obtained also for reactions between (1, 2e and 3) and (1, 2d and 3).

The experimental results indicate that the third step (rate constant $k_{3}$ ) is possibly fast. In contrast, it may be assumed that the third step is the rate determining step for the proposed mechanism. In this case the rate law can be expressed as follows:

rate $=k_{3}\left[I_{1}\right][3]$

The steady state assumption can be employed for $\left[I_{1}\right]$ which is generated following equation,

$\left[I_{1}\right]=\frac{k_{2}[1][2]}{k_{-2}+k_{3}[3]}$

The value of $\left[\mathrm{I}_{1}\right]$ can be replaced in equation (3) to obtain this Eq.

rate $=\frac{k_{2} k_{3}[1][2][3]}{k_{-2}+k_{3}[3]}$

Since it was assumed that $k_{3}$ is relevant to the rate determining step, it is reasonable to make the following assumption:

$k_{-2}>k_{3}[3]$

So the rate of low becomes:

rate $=\frac{k_{2} k_{3}[1][2][3]}{k_{-2}}$

The final equation is indicates that overall order of reaction is three which is not compatible with experimental overall order of reaction $(=2)$. In addition, according to this equation, the order of reaction with respect to $\mathrm{NH}$-acid 3 is one, whereas it was actually shown to be equal to zero. For this reason, it appeared that the third step is fast. If we assume that the fourth step (rate constant $k_{4}$ ) is the rate-determining step for the proposed mechanism, in this case, there are two ionic species to consider in the rate determining step, namely phosphonium ion $\left(I_{2}\right)$ and benzotriazole ion $\left(\mathrm{Z}^{-}\right)$. The phosphonium and benzotriazole ions, as we see in Fig. 8, have full positive and negative charges and form very powerful ion-dipole bonds to the 1,2-dichloroethane, the high dielectric constant solvent. However, the transition state for the reaction between two ions carries a dispersed charge, which here is divided between the attacking benzotriazole and the phosphonium ions. Bonding of solvent (1,2dichloroethane) to this dispersed charge would be much weaker than to the concentrated charge of benzotriazole and phosphonium ions. The solvent thus stabilize the species ions more than it would the transition state, and therefore $\mathrm{E}_{\mathrm{a}}$ would be higher, slowing down the reaction. However, in practice, 1,2-dichloroethane speeds up the reaction and for this reason, the fourth step, which is independent of the change in the solvent medium, could not be the rate determining step. Furthermore, the rate law of formation of the product (fourth step) for a proposed reaction mechanism with application of steady state assumption can be expressed by:

rate $=k_{4}\left[I_{2}\right]\left[Z^{-}\right]$

By application of steady state for $\left[\mathrm{I}_{2}\right]$ and $\left[\mathrm{Z}^{-}\right]$, and replacement of their values in the above equation, the following equation is obtained: 
rate $=\frac{k_{2} k_{3}[1][2][3]}{k_{-2}+k_{3}[3]}$

This equation is independent of rate constant for the fourth step $\left(k_{4}\right)$ and shows why the fourth step would not be affected by a change in the solvent medium. In addition, it has been suggested earlier that the kinetics of ionic species phenomena (e.g., the fourth step) are very fast. If the first step (rate constant $k_{2}$ ) were the rate determining step, in this case, two reactants (triphenylphosphine $\mathbf{1}$ and dialkyl acetylenedicarboxylate 2), as we see in Fig. 8, have no charge and could not form strong iondipole bonds to the high dielectric constant solvent, 1, 2-dichloroethane. However, the transition state carries a dispersed charge which here is divided between the attacking $\mathbf{1}$ and $\mathbf{2}$ and, hence, bonding of solvent to this dispersed charge is much stronger than the reactants, which lack charge. The solvent thus stabilizes the transition state more than it does the reactants and, therefore, $\mathrm{E}_{\mathrm{a}}$ is reduced which speeds up the reaction. Our experimental results show that the solvent with higher dielectric constant exerts a power full effect on the rate of reaction (in fact, the first step has rate constant $k_{2}$ in the proposed mechanism) but the opposite occurs with the solvent of lower dielectric constant, (see Table 1). The results of the current work (effects of solvent and concentration of compounds) have provided useful evidence for steps $1\left(k_{2}\right), 3\left(k_{3}\right)$ and $4\left(k_{4}\right)$ of the reactions between triphenylphosphine $\mathbf{1}$, dialkyl acetylenedicarboxylate $\mathbf{2}$ (2d, $\mathbf{2 e}$ or $\mathbf{2 f}$ ) and $\mathrm{NH}$-acid $\mathbf{3}$ (3a, 3b or 3c). Two steps involving 3 and 4 are not determining, although the discussed effects, taken altogether, are compatible with first step $\left(k_{2}\right)$ of the proposed mechanism and would allow it to be the rate-determining step. However, a good kinetic description of the experimental result using a mechanistic scheme based upon the steady state approximation is frequently taken as evidence of its validity. By application of this, the rate formation of product $\mathbf{4}$ from the reaction mechanism (Fig. 8) is given by:

$\frac{d[4]}{d t}=\frac{d[\text { ylide }]}{d t}=$ rate $=k_{4}\left[I_{2}\right]\left[Z^{-}\right]$

We can apply the steady-state approximation to $\left[\mathrm{I}_{1}\right]$ and $\left[\mathrm{I}_{2}\right]$;

$$
\begin{gathered}
\frac{d\left[I_{1}\right]}{d t}=k_{2}[1][2]-k_{-2}\left[I_{1}\right]-k_{3}\left[I_{1}\right][3], \\
\frac{d\left[I_{2}\right]}{d t}=k_{3}\left[I_{1}\right][3]-k_{4}\left[I_{2}\right]\left[Z^{-}\right]
\end{gathered}
$$

To obtain a suitable expression for $\left[\mathrm{I}_{2}\right]$ to put into equation (5) we can assume that, after an initial brief period, the concentration of $\left[I_{1}\right]$ and $\left[I_{2}\right]$ achieve a steady state with their rates of formation and rates of disappearance just balanced. Therefore $\frac{d\left[I_{1}\right]}{d t}$ and $\frac{d\left[I_{2}\right]}{d t}$ are zero and we can obtain expressions for $\left[I_{2}\right]$ and $\left[I_{1}\right]$ as follows:

$$
\begin{array}{lr}
\frac{d\left[I_{2}\right]}{d t}=0 & \quad\left[I_{2}\right]=\frac{k_{3}\left[I_{1}\right][3]}{k_{4}\left[Z^{-}\right]} \\
\frac{d\left[I_{1}\right]}{d t}=0, & {\left[I_{1}\right]=\frac{k_{2}[1][2]}{k_{-2}+k_{3}[3]}}
\end{array}
$$

We can now replace $\left[I_{1}\right]$ in the Eq. (6) to obtain this equation:

$\left[I_{2}\right]=\frac{k_{2} k_{3}[1][2][3]}{k_{4}\left[Z^{-}\right]\left[k_{-2}+k_{3}[3]\right]}$

The value of $\left[\mathrm{I}_{2}\right]$ can be put into equation (5) to obtain the rate Eq. (8) for proposed mechanism:

$$
\text { rate }=\frac{k_{2} k_{3} k_{4}[1][2][3]\left[Z^{-}\right]}{k_{4}\left[Z^{-}\right]\left[k_{-2}+k_{3}[3]\right]} \quad \text { or } \quad \text { rate }=\frac{k_{2} k_{3}[1][2][3]}{\left[k_{-2}+k_{3}[3]\right]}
$$


Since experimental data were indicated that steps $3\left(k_{3}\right)$ and $4\left(k_{4}\right)$ are fast but step $1\left(k_{2}\right)$ is slow, it is therefore reasonable to make the following assumption:

$$
k_{3}[3]>>k_{-2}
$$

So the rate equation becomes:

rate $=k_{2}[1][2]$

This equation which was obtained from a mechanistic scheme (shown in Fig. 8) by applying the steady-state approximation is compatible with the results obtained by UV spectrophotometery. With respect to the Eq. (9) that is overall reaction rate, the activation parameters involving $\Delta G^{\#}, \Delta S^{\#}$ and $\Delta \mathrm{H}^{\#}$ could be now calculated for the first step (rate determining step), as an elementary reaction, on the basis of Eyring equation. The results are reported in Table 4.

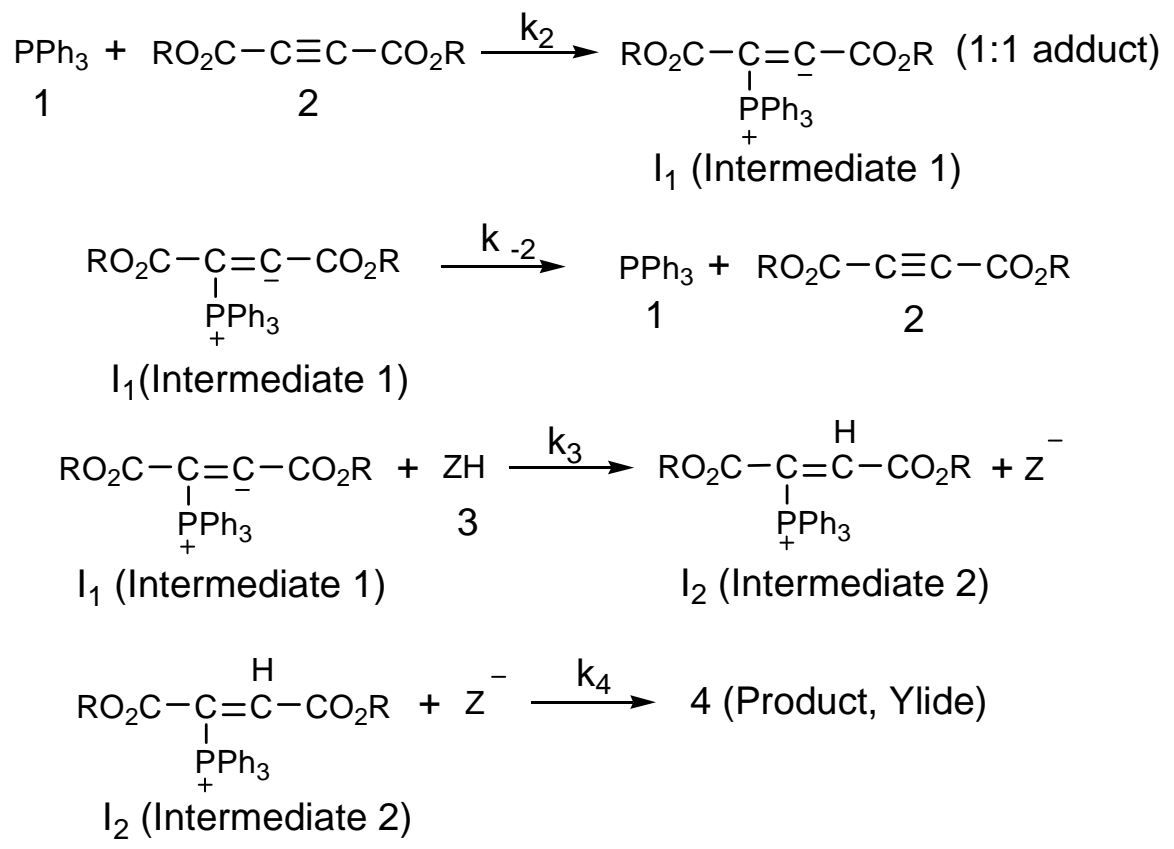<smiles></smiles>

Fig. 8. Mechanism proposed for the reaction between $\mathbf{1}, \mathbf{2}(\mathbf{2 d}, \mathbf{2 e}$ or $\mathbf{2 f})$ and $\mathbf{3}(\mathbf{3 a}, \mathbf{3 b}$ or $\mathbf{3 c})$ on the basis of literatures for generation of phosphorus ylides 4 .

\subsection{Further kinetic investigations}

\subsubsection{Effect of structure of dialkyl acetylenedicarboxylates}

To confirm the above observations, further experiments were performed with diethyl acetylenedicarboxylate $\mathbf{2 e}$ and dimethyl acetylenedicarboxylate $\mathbf{2 d}$, respectively, under the same conditions used in the previous experiments. The values of the second-order rate constant $\left(k_{2}\right)$ for the reactions between $(\mathbf{1}, \mathbf{2 e}$ and $\mathbf{3})$ and $(\mathbf{1}, \mathbf{2 d}$ and $\mathbf{3})$ are reported in Table $\mathbf{2}$, for all solvents and temperatures investigated. 
Table 2 The values of second order rate constant for the reaction between $\mathbf{1}, \mathbf{2 e}$ and $\mathbf{3}$ in the presence of different solvents at all temperatures investigated

\begin{tabular}{|c|c|c|c|c|c|c|}
\hline \multirow{2}{*}{3} & \multirow{2}{*}{ Solvent } & \multirow{2}{*}{$\varepsilon$} & \multicolumn{4}{|c|}{$k_{2} \cdot \mathbf{M}^{-1} \cdot \min ^{-1}$} \\
\hline & & & $12.0^{\circ} \mathrm{C}$ & $17.0^{\circ} \mathrm{C}$ & $22.0^{\circ} \mathrm{C}$ & $27.0^{\circ} \mathrm{C}$ \\
\hline \multirow{2}{*}{ За } & 1,4-dioxan & 2.02 & 377.2 & 418.6 & 492.1 & 612.4 \\
\hline & 1,2-dichloroethane & 10.3 & 521.4 & 653.0 & 854.1 & 1036.2 \\
\hline \multirow{2}{*}{$3 \mathbf{b}$} & 1,4-dioxan & 2.02 & 278.6 & 297.4 & 356.5 & 458.3 \\
\hline & 1,2-dichloroethane & 10.3 & 475.6 & 569.2 & 659.4 & 848.2 \\
\hline \multirow{2}{*}{ 3c } & 1,4-dioxan & 2.02 & 491.8 & 544.2 & 598.6 & 713.2 \\
\hline & 1,2-dichloroethane & 10.3 & 635.2 & 780.1 & 96.0 & 1163.5 \\
\hline
\end{tabular}

Table 3 The values of second order rate constant for the reaction between $\mathbf{1}, \mathbf{2} \mathbf{d}$ and $\mathbf{3}$ in the presence of different solvents at all temperatures investigated

\begin{tabular}{|c|c|c|c|c|c|c|}
\hline \multirow{2}{*}{3} & \multirow{2}{*}{ Solvent } & \multirow{2}{*}{$\varepsilon$} & \multicolumn{4}{|c|}{$k_{2} \cdot \mathbf{M}^{-1} \cdot \min ^{-1}$} \\
\hline & & & $12.0^{\circ} \mathrm{C}$ & $17.0^{\circ} \mathrm{C}$ & $22.0^{\circ} \mathrm{C}$ & $27.0^{\circ} \mathrm{C}$ \\
\hline \multirow{2}{*}{$3 a$} & 1,4-dioxan & 2.02 & 408.1 & 476.3 & 546.6 & 625.2 \\
\hline & 1,2-dichloroethane & 10.3 & 626.9 & 742.3 & 931.8 & 1191.6 \\
\hline \multirow{2}{*}{$3 \mathbf{b}$} & 1,4-dioxan & 2.02 & 381.7 & 431.3 & 502.1 & 599.7 \\
\hline & 1,2-dichloroethane & 10.3 & 531.2 & 623.1 & 721.0 & 851.4 \\
\hline \multirow{2}{*}{$3 c$} & 1,4-dioxan & 2.02 & 577.0 & 621.1 & 687.9 & 783.8 \\
\hline & 1,2-dichloroethane & 10.3 & 712.5 & 855.1 & 1045.3 & 1295.7 \\
\hline
\end{tabular}

Table 4 The activation parameters involving $\Delta \mathrm{G}^{\#}, \Delta \mathrm{S}^{\#}$ and $\Delta \mathrm{H}^{\#}$ for the reactions between $(\mathbf{1}, \mathbf{2} \mathbf{f}$ and 3a), (1, 2f and 3b) and (1, 2f and 3c) in 1,4-dioxan

\begin{tabular}{ccccc}
\hline Reactions & $\mathrm{Ea}\left(\mathrm{kJ} \cdot \mathrm{mol}^{-1}\right)$ & $\Delta \mathrm{G}^{\#}\left(\mathrm{~kJ} \cdot \mathrm{mol}^{-1}\right)$ & $\Delta \mathrm{H}^{\#}\left(\mathrm{~kJ} \cdot \mathrm{mol}^{-1}\right)$ & $\Delta \mathrm{S}^{\#}\left(\mathrm{~J}^{-1} \mathrm{~mol}^{-1} \cdot \mathrm{K}^{-1}\right)$ \\
\hline 1, 2f and 3a & 32.2 & 69.8 & 29.8 & -136.7 \\
1, 2f and 3b & 33.6 & 70.0 & 31.2 & -132.7 \\
1, 2f and 3c & 26.5 & 69.6 & 24.1 & -155.6 \\
\hline
\end{tabular}

As can be seen from Tables 2 and 3 the behavior of diethyl acetylenedicarboxylate $\mathbf{2 e}$ and dimethyl acetylenedicarboxylate $\mathbf{2 d}$ is the same as for the di-tert-butyl acetylenedicarboxylate $\mathbf{2 f}$ with respect to the reaction with triphenylphosphine $\mathbf{1}$ and $\mathrm{NH}$-acid $\mathbf{3}$ (3a, 3b and 3c). The rate of the former reactions was also accelerated in a higher dielectric constant environment and with higher temperatures; however, these rates under the same condition are approximately 4 to 7 times more than for the reaction with di-tert-butyl acetylenedicarboxylate $2 \mathbf{f}$. It seems that both inductive and steric factors for the bulky alkyl groups in $\mathbf{2 f}$ tend to reduce the overall reaction rate (see equation 9). In the case of dimetyl acetylenedicarboxylate 2d, the lower steric and inductive effects of the dimethyl groups exert a powerful effect on the rate of reaction.

\subsubsection{Effect of structure of $\mathrm{NH}$-acids (substituted group on the ring of benzotriazole}

Reproducible experiments were investigated in the presence of other $\mathrm{NH}$-acids such as 5methylbenzotriazole $\mathbf{3 b}$ or 5-chlorobenzotriazole $\mathbf{3 c}$ under the same conditions used in the previous experiments, the rate of reactions were reduced in comparison with benzotriazole 3a respectively. It seems that the effect of electron donor of substituted methyl groups in position 5 of benzotriazole ring would tend to reduce the chemical activity of $\mathrm{NH}$-acid, conversely electron withdrawal of halogen 
group in the same position would tend to increase the chemical activity of NH-acid in comparison with benzotriazole 3a (see Tables 1, 2 and 3). Practically, experimental data were indicate that change within the structure of $\mathrm{NH}$-acid has no effect on the priority of the rate determining step of the reaction and step 1 (rate constant $k_{2}$ ) is still the rate determining step, in the presence of new NHacids $3 \mathbf{c}$ or $\mathbf{3 b}$ and order of reaction with respect to them is still zero. Because of the chemical activity of $\mathrm{NH}$-acid is dependent on its structure, this provide opportunity in order to change the rate constant $k_{3}$ (NH-acid participate in this step) and also subsequent alteration of overall rate constant of reaction (see equation 8).

\section{Conclusions}

Kinetic investigation of these reactions was undertaken using UV spectrophotometry. The results can be summarized as follow: (1) the appropriate wavelengths and concentrations were determined to follow the reaction kinetics. (2) The overall reaction order followed second-order kinetics and the reaction orders with respect to triphenylphosphine, dialkyl acetylenedicarboxylate and $\mathrm{NH}$-acid were one, one and zero respectively. (3) The values of the second-order rate constants of all reactions were calculated automatically with respect to the standard equation, using the software associated with the Cary-300 UV equipment. (4) The rates of all reactions were accelerated at higher temperatures. Under the same conditions, the activation energy for the reaction in the presence of benzotriazole 3a $(32.2 \mathrm{~kJ} / \mathrm{mol})$ was higher than that for the both reactions which were followed by the 5methylbenzotriazole $3 \mathbf{b}(33.6 \mathrm{~kJ} / \mathrm{mol})$ and 5-chlorobenzotriazole 3c $(26.5 \mathrm{~kJ} / \mathrm{mol})$ in 1,4-dioxan (5) The rates of all reactions were increased in solvents of higher dielectric constant and this can be related to differences in stabilization by the solvent of the reactants and the activated complex in the transition state. (6) Increased steric bulk in the alkyl groups of the dialkyl acetylenedicarboxylates, accompanied by the correspondingly greater inductive effect, reduced the overall reaction rate. (7) With respect to the experimental data, the first step of proposed mechanism was recognized as a ratedetermining step $\left(k_{2}\right)$ and this was confirmed based upon the steady-state approximation. (8) Also, the third step was identified as a fast step $\left(k_{3}\right)$. (9) The activation parameters involving $\Delta \mathrm{G}^{\#}, \Delta \mathrm{S}^{\#}$ and $\Delta \mathrm{H}^{\#}$ were reported for three reactions.

\section{Acknowledgments}

Authors sincerely thank the University of Sistan \& Baluchestan and Payame Noor University for providing financial support of this work.

\section{References}

1. Crayson M., and Griffith E. J. (1972) Topics in Phosphorus Chemistry, vol. 7. Insterscience, New York.

2. Hudson H. R. (1990) Primary, Secondary and Tertiary Phosphines, Plyphosphines and Heterocyclic Organophosphorus Compounds, in the Chemistry of Organophosphorus Compounds. Wiley: New York, 1, 386-472.

3. Engel R. (1988) Synthesis of Carbon-phosphorus Bonds. CRC Press: Boca Rotan, FL, 1988.

4. Cadogan J. I. G. (1979) Organophosphorus Reagent in Organic Synthesis, Academic press: New York.

5. Ramazani A., Noshiranzadeh N., and Ghamkhari A., (2008) One-pot diastereoselective synthesis of densely functionalized $2-\mathrm{H}$ indeno [2,1-b] furans. Single-crystal X-ray structure of dimethyl 8,8a-dihydro-8-oxo-8a-(2,2,2-trichloroethoxy)-2H-indeno [2,1-b] furan- 2,3dicarboxylate. Helv. Chim. Acta., 91, 2252-2261.

6. Ramazani A., Kazemizadeh A. R., Ahmadi E., Noshiranzadeh N., and Souldozi A. (2001) Synthesis and reactions of stabilized phosphorus ylides. Curr. Org. Chem., 2, 59-82.

7. Maryanoff B. E., and Reitz A. B. (1989) The Wittig olefination reaction and modifications involving phosphoryl-stabilized carbanions. Stereochemistry, mechanism, and selected synthetic aspects. Chem. Rev., 89, 863-927. 
8. Maghsoodlou M. T., Hazeri N., Habibi-Khorassani S. M., Moeeni Z., Marandi Gh., Lashkari M., Ghasemzadeh M., and Bijanzadeh H. R. (2007) Water-acetone media enforced chemoselective synthesis of 2-substituted pyrrole stable phosphorus ylides from reaction between pyrrole and acetylenic esters in the presence of triphenylphosphine. J. Chem. Res., 566-568.

9. Anary-Abbasinejad M., Anaraki-Ardakani H., and Hosseini-Mehdiabad H. (2008) One-pot synthesis of stable phosphorus ylides by three-component reaction between dimethyl acetylenedicarboxylate, semicarbazones, and triphenylphosphine. Phosphorus Sulfur and Silicon, 183, 1440-1446.

10. Hassanabadi A., Anary-Abbasinejad M., and Dehghan A. (2009) Three-component reaction of triphenylphosphine, dimethyl acetylenedicarboxylate, and aldehyde benzoylhydrazones: an efficient one-pot synthesis of stable phosphorus ylides. Synth. Commun., 39, 132-138.

11. Anaraki-Ardakani H., Sadeghian Sh., Rastegari F., Hassanabadi A., and Anary-Abbasinejad M. (2008) Three-component reaction of triphenylphosphine, acetylenic esters, and arylsulfonyl hydrazines: an efficient one-pot synthesis of stable $\beta$ - nitrogen-substituted phosphourus ylides. Synth. Commun., 38, 1990-1999.

12. Yavari I., Adib M., Jahani-Mogaddam F., and Sayahi M. H. (2002) A simple synthesis of stable heterocyclic phosphorus ylides derived from NH-acids. Phosphorus Sulfur and Silicon, $177,545-553$.

13. Kazemian M. A., Nassiri M., Ebrahimi A., Maghsoodlou M. T., and Habibi-Khorassani S. M. (2008) A facile synthesis, dynamic ${ }^{1} \mathrm{H}$ NMR, and theoretical study of novel stable heterocyclic phosphorus ylides containing a tetrazole ring. Arkivoc, (xvii), 173-183.

14. Fitjer L., and Quabeck U. (1985) The Wittig reaction using potassium-tert-butoxide high yield methylenations of sterically hindered ketones. Synth. Commun., 15, 855-864.

15. Ramazani A., and Souldozi A. (2005) Dipotassium-hydrogen-phosphate-powder-catalyzed stereoselective C-vinylation of diphenylacetonitril. Phosphorus Sulfur Silicon, 180, 28012804.

16. Yavari I., and Alizadeh A. A. (2003) A simple approach to the synthesis of 1, 4-bis (arylsulfonyl) tetrahydropyrazine-2, 5-diones. Monatsh. Chem., 134, 435-438.

17. Ramazani A., and Bodaghi A. (2000) One-pot, four-component synthesis of dialkyl [indane-1, 3-dione-2-ylidene] alkoxysuccinates. Tetrahedron Lett., 41, 567-568.

18. Wang Z. G., Zhang G. T., Guzei I., and Verkade J. G. (2001) $\mathrm{PhCH}=\mathrm{P}\left(\mathrm{MeNCH}_{2} \mathrm{CH}_{2}\right)_{3} \mathrm{~N}$ : A novel ylide for quantitative $E$ selectivity in the wittig reaction. J. Org. Chem., 66, 3521-3524.

19. Islami M. R., Hassani Z., and Saidi K. (2003) An efficient and rapid one-pot synthesis of highly functionalized stable phosphorus ylides containing the sulfur atom. Synth. Commun., $33,65-72$.

20. Keglevich G., Baan Z., and Hermecz I. (2007) The phosphorus aspects of green chemistry: the use of quaternary phosphonium salts and 1,3-dialkylimidazolium hexafluorophosphates in organic synthesis. Curr. Org. Chem., 11, 107-126.

21. Yavari, I., and Ahmadian-Rezlighi L. (2006) Triphenylphosphine-mediated reaction between dimethyl acetylenedicarboxylate and NH-acids derived from diaminobenzenes. Phosphorus Sulfur and Silicon, 181, 771-777.

22. Adib M., Mostofi M., Ghanbary K., and Bijanzadeh H. R. (2005) Vinyltriphenylphosphonium salt-mediated efficient synthesis of iminophosphoranes derived from 2-aminothiazoles. Synthesis, 10, 1663-1668.

23. Kalantari M., Islami M. R., Hassani Z., and Saidi K. (2006) Synthesis of dimethyl-1(trifluoromethyl)-3H-pyrrolizine-2, 3-dicarboxylate using phosphorus compounds. Arkivoc, (x), 55-62.

24. Islami M. R., Mollazehi F., Badiei A., and Sheibani H. (2005) Serendipitous synthesis of 1, 4benzothiazin derivatives using 2-[(2-aminophenyl)disulfanyl aniline, Arkivoc, (xv), 25-29. 
25. Maghsoodlou M. T., Habibi-Khorassani S. M., Rofouei M. K., Adhamdoust S. R., and Nassiri M. (2006) Triphenylphosphite as a good reagent for the diasteroselective synthesis of phosphonate esters. Arkivoc, (xii), 145-151.

26. Maghsoodlou M. T., Hazeri N., Habibi-Khorassani S. M., Ghulame-Shahzadeh A., and Nassiri M. (2006) A simple synthesis of stable phosphorus ylides from indole and some of its derivatives. Phosphorus Sulfur and Silicon, 181, 913-919.

27. Habibi-Khorassani S. M., Maghsoodlou M. T., Ebrahimi A., Zakarianejad M., and Fattahi M. (2007) Kinetics and mechanism of the reactions between triphenylphosphine, dialkyl acetylenedicarboxilates and a $\mathrm{NH}$-acid, pyrazole, by UV spectrophotometry. J. Soln. Chem., $36,1117-1127$.

28. Sasaki S., and Yoshifuji M. (2001) Synthesis, structure and properties of crowded triarylphosphines. Curr. Org. Chem., 11, 17-31.

29. Habibi-Khorassani S. M., Maghsoodlou M. T., Nassiri M., Zakarianejad M., and Fattahi M. (2006) Synthesis of stable phosphorus ylides from 3, 5-dimethylpyrazoleand kinetic investigation of the reactions by UV spectrophotometry. Arkivoc, (xvi), 168-184.

30. Maghsoodlou M. T., Habibi Khorassani S. M., Hazeri, N., Kakaei, R., Nassiri, M., and Marandi G. (2006). An efficient synthesis of stable phosphorus ylides derived from triphenylphosphine, dialkyl acetylenedicarboxylates, and an NH-Acid. Phosphorus, Sulfur and Silicon, 181, 4, 865-877.

31. Schwartz L. M., and Gelb R. I. (1978) Alternative method of analyzing first-order kinetic data. Anal. Chem. 50, 11, 1592-1594. 